\title{
Autoeficácia e motivação na resolução de problemas matemáticos
}

\section{contextualizados}

\section{Self-efficacy and motivation in solving contextualized mathematical problems}

\author{
Amanda Pranke, Lourdes Maria Bragagnolo Frison \\ Universidade Federal de Pelotas
}

\begin{abstract}
Resumo
Este trabalho está alicerçado na autorregulação da aprendizagem e objetivou aferir as crenças de autoeficácia sobre a resolução de problemas propostos em entrevistas com tarefa, realizadas com seis estudantes de Ensino Fundamental de uma escola agrícola, identificando também, as fontes da autoeficácia deles no decorrer da pesquisa. Analisamos uma das quatro entrevistas com tarefa realizadas com os estudantes, o que revelou que eles possuem elevadas crenças de autoeficácia para resolverem problemas, obtendo resultados positivos e as fontes das crenças de autoeficácia deles envolvem experiências de êxito e experiências vicárias associadas à resolução de problemas no contexto agrícola.

Palavras-chave: Autorregulação da aprendizagem, autoeficácia, dimensão contextual, problemas matemáticos.
\end{abstract}

\begin{abstract}
This work is based on self-regulation of learning and aims to assess the beliefs of self-efficacy on the resolution of problems proposed in interviews with task, carried out with six elementary school students of an agricultural school, also identifying the sources of their self-efficacy in the course of the research. We analyzed one of the four task interviews conducted with the students, which revealed that they have high self-efficacy beliefs to solve problems, obtaining positive results and the sources of their self-efficacy beliefs involve successful experiences and vicarious experiences associated with problem solving in the agricultural context.

Keywords: Self-regulation of learning, self-efficacy, contextual dimension, mathematical problems.
\end{abstract}

A autorregulação da aprendizagem alicerça este estudo, por ser entendida como um processo que estimula os estudantes a definirem metas e a desenvolverem estratégias com as quais buscam alcançar resultados positivos na resolução de problemas (Zimmerman, 2000; 2013; Zimmerman; Campillo, 2003). A autorregulação da aprendizagem é compreendida como um processo multidimensional, que envolve as dimensões: cognitiva/metacognitiva, motivacional, comportamental e contextual (Veiga Simão, 2006). No presente estudo teorizamos sobre a dimensão motivacional e, mais especificamente, sobre as crenças de autoeficácia.
Ao estudarmos sobre motivação, podemos dizer que ela é o processo pelo qual se iniciam e se apoiam as atividades orientadas para os objetivos. A motivação para aprender pode ser entendida como o que ativa, orienta e mantém a decisão de estudar, sendo esta diferente de estudante para estudante. A motivação corresponde à energia para iniciar e manter uma ação, fazendo com que o indivíduo persista nela. Sendo assim, a motivação é fator desencadeante para que o estudante autorregule a sua aprendizagem (Rosário; Núñez; González-Pienda, 2006; Rosário Et Al., 2008; Souza, 2010; Pereira, 2013).

No contexto escolar, o estudante se motiva para aprender se os conteúdos lhe fizerem sentido, assim se mobilizará a buscar, a pesquisar, aguçando sua curiosidade, tornando a aprendizagem ativa e dinâmica (Bzuneck, 2009). A motivação, segundo Lopes da Silva (2004), contribui para que o sujeito defina objetivos que promovam aprendizagem, portanto ela está associada a aspectos da ativação e sustentação dos comportamentos dirigidos para a aprendizagem. Em relação aos processos de ensino e de aprendizagem, a motivação tem uma pertinência relevante porque, para aprender, os estudantes precisam estar cognitiva, emocional e comportamentalmente envolvidos nas atividades escolares. Só dessa forma as atividades podem ser úteis e produtivas (Pereira, 2013).

Remetendo-nos à compreensão sobre a importância da motivação nas aulas de Matemática, percebemos com os estudos de Sousa et al. (2010) e Ricardo et al. (2012) que os estudantes com melhor desempenho em Matemática apresentaram níveis motivacionais mais elevados. Assim, inferimos que os estudantes, ao utilizarem estratégias que aumentem seus níveis motivacionais, poderão melhorar o desempenho em Matemática (Lazzari, 2009; Jesus, 2011; Nunes, 2011).

Considerando a dimensão motivacional é importante discutirmos as crenças de autoeficácia. A autoeficácia é compreendida como o julgamento a respeito da própria capacidade em cumprir tarefas específicas (Bandura, 1997). Essa crença não se refere especificamente à capacidade de um estudante, mas sim ao que ele acredita ser capaz de realizar em um contexto de aprendizagem, 
ou no caso dessa pesquisa, na resolução de problemas (Souza, 2006).

É interessante ressaltar que estudantes que possuem elevadas crenças de autoeficácia, estabelecem um elevado nível de motivação, o que garante um maior esforço e persistência diante das dificuldades, tendo interesse em cumprir a tarefa proposta pelo professor (Costa; Boruchovitch, 2006; Bzuneck, 2009; Azzi; Polydoro, 2010). Por exemplo, se o estudante se sente capaz de resolver um problema de Matemática, ele estará motivado para encontrar a solução e mesmo que tenha dificuldades se esforçará para resolver a tarefa com êxito.

Costa e Boruchovitch (2006) baseadas nos estudos de Bandura (1997) explicam que existem quatro fontes responsáveis por estabelecer a percepção de autoeficácia, que são: experiências de êxito, experiências vicárias, persuasão verbal e estados fisiológicos. A experiência de êxito é a fonte que exerce maior influência sobre as crenças de autoeficácia, pois representa experiências pessoais reais (Costa; Boruchovitch, 2006; Bzuneck, 2009; Azzi; Polydoro, 2010). Se o estudante obteve sucesso repetidas vezes ao resolver problemas de Matemática, é provável que ele se sinta mais confiante em relação as suas capacidades, o que poderá fortalecer seu senso de autoeficácia. Da mesma forma, se o estudante sempre encontra muitas dificuldades em Matemática, suas crenças de autoeficácia podem ser afetadas negativamente.

As experiências vicárias envolvem a observação de modelos sociais, ou seja, ver pessoas com capacidades análogas desempenhando tarefas com sucesso contribui para que o estudante também se sinta capaz de realizar determinada tarefa (Costa; Boruchovitch, 2006; Bzuneck, 2009). No ambiente escolar, o estudante ao observar os colegas resolvendo corretamente um problema de Matemática, tende a se sentir capaz de também conseguir resolver.

A persuasão verbal é caracterizada pelas informações que os estudantes recebem do professor, por exemplo, sobre suas capacidades ou desempenho em determinadas tarefas (Souza, 2006; Bzuneck, 2009). Os estudos de Shih e Alexander (2000) apontaram que o tipo de feedback oferecido ao estudante tem efeito sobre sua autoeficácia, por exemplo, foi mais positivo aquele feedback que comparou o rendimento atual do estudante com desempenhos anteriores do que o feedback que comparou o desempenho do estudante com os demais colegas.

Por fim, outra fonte que afeta as crenças de autoeficácia do estudante são as reações fisiológicas. Quanto mais calmo o estudante estiver mais controle sobre seu raciocínio ele terá, elevando suas crenças de autoeficácia (Costa; Boruchovitch, 2006; Azzi; Polydoro, 2010). Por exemplo, se o estudante ficar muito ansioso na prova de Matemática, poderá começar a suar e ter seus batimentos cardíacos acelerados, essas reações fisiológicas podem influenciar as expectativas do estudante quanto a se sair bem na prova.

Nas últimas décadas, as crenças de autoeficácia têm sido foco de muitas investigações, relacionadas ao bom desempenho em Matemática. Autores como, Schunk (1998); Rosário et al. (2008); Souza; Brito (2008);
Dobarro; Brito (2010); Torisu (2010; 2012); Souza (2013); Machado (2013); Brito; Souza (2015), demonstraram em seus estudos, que existe uma relação altamente significativa entre o desempenho, a atitude e a autoeficácia em relação à Matemática.

Existe, conforme Pintrich (1994; 2000), uma nítida correlação entre cognição, motivação e contexto. Para o autor a partir da cognição e da motivação podem ser criadas metas e objetivos para atingir resultados, desde que se tenha um contexto propício à aprendizagem, ou por meio de uma tarefa que estimule o estudante a pesquisar e buscar estratégias de resolução para os problemas propostos.

Inferimos que os elementos motivacionais e cognitivos se encontram em conexão, tendo em conta o contexto no qual ocorre a aprendizagem e, nessa perspectiva, tanto os processos motivacionais quanto os cognitivos podem ser regulados pelo próprio estudante. No momento em que ele se motiva para estudar, utiliza estratégias específicas para resolver um problema e, se necessário, busca ajuda para conseguir atingir seu objetivo de aprendizagem, ele está apresentando características de um comportamento autorregulado.

A partir do que foi exposto, o objetivo deste trabalho foi aferir as crenças de autoeficácia sobre a resolução de problemas propostos em entrevistas com tarefa, realizadas com seis estudantes de Ensino Fundamental de uma escola agrícola, identificando também, as fontes da autoeficácia deles no decorrer da pesquisa.

\section{Processo metodológico da pesquisa}

Este estudo de abordagem qualitativa se classifica como um estudo de caso (Yin, 2010), realizado com seis estudantes de uma turma dos anos finais do Ensino Fundamental de uma escola agrícola do sul do Brasil. Os participantes têm, atualmente, 14 anos de idade, sendo quatro do sexo masculino e dois do sexo feminino, todos filhos de agricultores, residentes nas proximidades da escola. Fizemos um acompanhamento da trajetória escolar dos estudantes durante três anos letivos consecutivos, do $6^{\circ}$ até o $8^{\circ}$ ano do Ensino Fundamental, ou seja, durante os anos de 2014, 2015 e 2016. A coleta de dados foi concluída ao final do ano de 2016, quando os estudantes cursavam o $8^{\circ}$ ano.

Para a coleta de dados utilizamos diferentes instrumentos, como por exemplo, um questionário, coletado em 2014, que objetivou mapear o perfil da turma e as características dos participantes; três observações em sala de aula e uma entrevista semiestruturada com cada estudante, realizadas em 2015, para identificar os conhecimentos do contexto, por eles mobilizados, ao resolverem problemas de Matemática; uma entrevista semiestruturada com dois agricultores, também em 2015, com o objetivo de coletar informações sobre os cálculos mais comuns realizados no meio agrícola e elaborar os problemas de Matemática com dados reais desse contexto. Por fim, em 2016, realizamos quatro entrevistas com tarefa com cada estudante, que se assemelham a uma entrevista semiestruturada e seguem um guia de questões, as quais os estudantes responderam antes e depois da resolução do problema de Matemática 
proposto. Durante o período de resolução do problema a pesquisadora observou o que o estudante fez, com a intenção de identificar o raciocínio matemático e as estratégias mobilizadas (Veiga Simão; Flores, 2007).

Dentre as tarefas propostas nas entrevistas, no decorrer da investigação, os estudantes resolveram problemas que versavam sobre aplicações de Matemática na produção de leite e no cultivo de tabaco, que caracterizam o trabalho de seus pais. Exemplificamos o que foi proposto apresentando aqui o problema resolvido pelos estudantes em uma das quatro entrevistas com tarefa.

Em uma propriedade rural, são utilizadas estufas elétricas e estufas convencionais para secar o fumo. $\mathrm{Na}$ estufa elétrica, o fumo demora 4 dias para secar e são gastos $3 m^{3}$ de lenha; já na estufa convencional, o fumo demora 7 dias para secar e é gasto o dobro de lenha. $O$ agricultor paga $R \$ 40,00$ por $1 \mathrm{~m}^{3}$ de lenha.

a) Qual o valor gasto na compra da lenha para cada estufa?

b) Explique ao produtor, com o auxílio de um gráfico, qual estufa é mais vantajosa.

Ao final da pesquisa, fizemos a transcrição e posteriormente, foi feita a análise textual discursiva (Moraes, 2003; Moraes; Galiazzi, 2007) da entrevista de onde emergiram as categorias de análise e os resultados do estudo. A análise dessa entrevista com tarefa é o foco de discussão do presente estudo.

\section{Discussão dos resultados da pesquisa}

A partir de uma leitura crítica e aprofundada dos depoimentos dos estudantes e fazendo articulações com o referencial teórico adotado nesse estudo, apresentamos duas categorias de análise: a) Elevadas crenças de autoeficácia sobre a resolução de problemas contextualizados e o bom desempenho em Matemática e b) Caraterização das fontes de autoeficácia sobre a resolução de problemas contextualizados. Essas categorias são originárias de unidades de significado que foram extraídas dos depoimentos dos estudantes participantes, exemplificados com as abreviações P1, P2, P3, P4, P5 e P6.

$\mathrm{Na}$ primeira categoria apresentamos os depoimentos dos estudantes sobre como percebiam o problema e como se sentiam frente à resolução. Destacamos o depoimento de P3 no qual afirma: "Acho esse problema fácil, parece que não pede muita coisa”. Da mesma forma P4 relata: "Eu acho esse problema bem simples, porque ele não tem muitos tipos de dados e contas" e P5 complementa: "Eu já sei como eu posso resolver, eu já tenho uma ideia assim bem fácil de como eu posso resolver e eu acho que vou conseguir resolver ele corretamente". A partir desses depoimentos percebemos a presença das crenças dos estudantes sobre suas capacidades para resolver o problema proposto, ou seja, o julgamento que os estudantes expressam sobre o que podem fazer com as habilidades que possuem (Bandura, 1997).

Percebemos que ambos os estudantes estavam familiarizados em resolver problemas contextualizados com o meio agrícola, o que os mantém com elevadas crenças de autoeficácia. Eles afirmam com segurança que acham o problema simples e que vão resolvê-lo com facilidade. Podemos inferir que a participação nessa pesquisa contribuiu para que esses estudantes se tornassem mais autorregulados para resolver esses problemas, porque um estudante autorregulado interpreta os novos problemas a partir de uma base que lhe seja familiar, ou seja, procura simplificar o problema apresentado se utilizando de seus conhecimentos prévios sobre o tema em questão (Rosário; Núñez; González-Pienda, 2006; Rosário, 2013).

Notamos que os estudantes apresentaram elevadas crenças de autoeficácia e tiveram facilidade em resolver os problemas contextualizados propostos na pesquisa, o que nos permitiu inferir que quanto mais motivado o estudante estiver e quanto mais elevadas forem suas crenças de autoeficácia, mais positivos serão seus resultados de aprendizagem, assim como também foi revelado em outros estudos (Dobarro; Brito, 2010; Brito; Souza, 2015).

$\mathrm{Na}$ segunda categoria procuramos revelar as fontes que contribuem para a percepção de autoeficácia dos estudantes sobre a resolução de problemas contextualizados. A partir de seus depoimentos percebemos que as fontes mais citadas foram às experiências de êxito e as experiências vicárias, enquanto que nenhum deles comentou sobre persuasão verbal e apenas P5 e P6 referiram-se às reações fisiológicas.

No que se refere às experiências de êxito, destacamos os depoimentos:

Eu acho esse problema fácil, porque eu já conheço essas contas, essas coisas (P2, 2016).

Como eu vivo em um ambiente agrícola isso me ajuda muito a entender o problema (...) em casa, logo que tinha acabado a secagem de uma estufa, meus pais pediram para eu fazer uma conta de quantos quilos dava de fumo, por exemplo, nós pesamos um grampo e depois contamos quantos grampos tinha dentro da estufa, para contar quantos quilos de fumo tinha dado em média a estufada (P5, 2016).

É um problema bem parecido, conhecido já, então já ajuda bastante e os conhecimentos que eu já tenho sobre esse tipo de problema também (P6, 2016).

Em relação às experiências vicárias, destacamos os depoimentos:

Em casa eu já vi o pai fazendo umas contas parecidas, quando nós empilhamos a lenha, nós vamos contando os metros (P2, 2016).

Eu vejo o pai e a mãe resolvendo contas como essa, aí eu já tenho uma noção de como fazer e saber se vai estar certo (P3, 2016).

Lá em casa são duas estufas elétricas que nós temos, meus pais fazem os cálculos para ver em quantos dias cada uma ia secar de diferença e para ver quantos dias ia levar até colher o fumo, quanto tempo levaria para começar a secar a estufa denovo, para as duas irem trabalhando juntas (P5, 2016).

Nós também compramos lenha então é meio parecido, meus pais fazem cálculos parecidos com esse (P6, 2016).

Já as reações fisiológicas foram citadas como fator positivo na resolução de problemas. Por exemplo, P5 menciona que a partir da pesquisa e dos encontros realizados mediante as entrevistas passou a se sentir mais calma e resolver melhor os problemas: "Essas 
entrevistas me ajudam a ter um pouco mais de segurança para resolver as contas e me ajudam a resolver cada parte, por cada etapa, eu era muito ansiosa". Por outro lado, P6 afirma que o fato de estar familiarizado com problemas contextualizados o deixa tranquilo e seguro durante a resolução: "Eu não fico nervoso para resolver esse tipo de problema, porque eu sei o que eu tenho que fazer, quais os passos corretos que eu tenho que seguir, ler o problema, resolver e depois corrigir".

Notamos nos depoimentos dos estudantes a presença marcante da dimensão contextual. As fontes das crenças de autoeficácia deles aparecem associadas ao contexto, tanto as experiências de êxito se remetem a problemas práticos que eles resolveram no contexto agrícola, quanto as experiências vicárias se referem ao fato de terem visto seus pais resolvendo problemas semelhantes. Além disso, eles afirmam que se sentem tranquilos e preparados para resolver problemas contextualizados, pois já sabem que estratégia poderá ser eficaz na resolução e ainda, sabem avaliar se a resposta está correta, pois a interpretam em um contexto real de aplicação.

É o contexto, segundo Knijnik et al. (2012), que constitui a referência para se compreender o significado das linguagens matemáticas, ou seja, os estudantes interpretaram os problemas tendo o seu próprio cotidiano como referência e modelo a ser utilizado. Além disso, os próprios pais são os modelos que os estudantes procuram seguir, eles os acompanham resolvendo problemas complexos no contexto agrícola e desejam também saber resolver aqueles problemas. Assim, esses modelos sociais competentes transmitem conhecimentos e ensinam estratégias eficientes para resolver problemas contextualizados, aumentando as crenças de autoeficácia dos estudantes (Costa; Boruchovitch, 2006).

\section{Conclusões}

Concluímos que os estudantes participantes da pesquisa possuem elevadas crenças de autoeficácia o que contribuiu para que tivessem um bom desempenho na resolução dos problemas de Matemática. Além disso, as fontes de autoeficácia apresentadas por eles são reforçadas pela valorização da dimensão contextual, uma vez que os estudantes possuem experiências positivas na resolução de problemas contextualizados com o meio agrícola e percebem seus pais como modelos competentes na resolução desse tipo de problema.

Esperamos como repercussões do estudo que atinjam o campo da aplicação prática, uma vez que acreditamos que trabalhos a respeito de atividades desenvolvidas no contexto escolar podem contribuir para os processos de aprendizagem dos estudantes envolvidos, além de mostrar aos professores os resultados positivos de um trabalho articulado ao contexto do estudante, no qual eles estavam motivados e mantendo elevadas suas crenças de autoeficácia.

\section{Referências}

Azzi, R. G. \& Polydoro, S. A. J. (2010). O papel da autoeficácia e autorregulação no processo motivacional. En: E. Boruchovitch, J. A. Bzuneck \& S.
E. R. Guimarães (Eds.). Motivação para aprender: aplicações no contexto educativo (pp. 126 - 144). Petrópolis, RJ: Vozes.

Bandura, A. (1997). Self-efficacy: the exercise of control. New York: W. H. Freeman.

Brito, M. R. F. \& Souza, L. F. N. I. (2015.). Autoeficácia na solução de problemas matemáticos e variáveis relacionadas. Temas em Psicologia, Ribeirão Preto, Vol. 23, p. 29-47.

Bzuneck, J. A. (2009). As crenças de autoeficácia e o seu papel na motivação do aluno. En: E. Boruchovitch \& J. A. Bzuneck (Eds.). A motivação do aluno: contribuições da Psicologia Contemporânea (pp.116 133). Petrópolis, RJ: Vozes.

Costa, E. R. \& Boruchovitch, E. (2006). A auto-eficácia e a motivação para aprender: considerações para o desempenho escolar dos alunos. En: R. G. Azzi \& S. A. J. Polydoro (Eds.). Auto-eficácia em diferentes contextos (pp. 87 - 109). Campinas, SP: Editora Alínea.

Dobarro, V. R. \& Brito, M. R. F. (2010). Atitude e crença de auto-eficácia: relações com o desempenho em Matemática. Educação Matemática e Pesquisa, São Paulo, No. 2, Vol. 12, p. 199-220.

JESUS, A. G. (2011). A motivação para aprender Matemática no $9^{\circ}$ ano do Ensino Fundamental: um estudo do potencial dos materiais manipulativos e da construção de objetos na aprendizagem de área de polígonos e volume de prismas. Dissertação (Mestrado em Educação Matemática) - Instituto de Ciências Exatas e Biológica, Universidade Federal de Ouro Preto, Ouro Preto.

Knijnik, G.; Wanderer, F.; Giongo, I. M.; \& Duarte, C. G. (2012). Etnomatemática em movimento. Belo Horizonte: Autêntica editora.

Lazzari, V. D. (2009). Matemática na agricultura: as práticas da agricultura motivando $\mathrm{o}$ ensino de matemática na $6^{\mathrm{a}}$ série. Trabalho referente à Pesquisa e Implementação na Escola (Programa de Desenvolvimento Educacional - PDE). Universidade Estadual do Oeste do Paraná, UNIOESTE, Paraná.

Lopes da Silva, A. (2004). A auto-regulação na aprendizagem: a demarcação de um campo de estudo e de intervenção. En: A. Lopes da Silva, A. M. Duarte, I. Sá \& A. M. Veiga Simão (Eds.). Aprendizagem auto-regulada pelo estudante: perspectivas psicológicas e educacionais (pp. 17 - 39). Porto: Porto Editora.

Machado, M. C. (2003). Um estudo sobre as atitudes em relação à matemática, crenças de autoeficácia matemática e o desempenho escolar dos estudantes. En: Atas do Encontro Nacional de Educação Matemática (pp. 1 - 14). Curitiba, Paraná.

Moraes, R. (2003). Uma tempestade de luz: a compreensão possibilitada pela análise textual discursiva. Ciência \& Educação, São Paulo, No. 2, Vol. 9, p. 191-211.

Moraes, R. \& Galiazzi, M. C. (2007). Análise Textual Discursiva. Ijuí: Ed. Unijuí

Nunes, E. A. A. C. (2011). Contributo das TIC na motivação para a aprendizagem da Matemática nos CEF do $3^{\circ}$ ciclo do ensino básico. Dissertação 
(Mestrado em Tecnologias e Metodologias em E-Learning) - Faculdade de Ciências, Universidade de Lisboa, Lisboa.

Pereira, A. (2013). Motivação na aprendizagem e no ensino. En: F. H. Veiga (Ed.). Psicologia da Educação: teoria, investigação e aplicação. Envolvimento dos alunos na escola (pp. 445 - 493). Lisboa: Climepsi Editores.

Pintrich, P. R. (1994). Student motivation in the college classroom. En: K. W. Prichard \& R. M. Sawyer (Eds.). Handbook of college teaching: theory and applications (pp. 23 - 43). Westport, CT: Greenwood.

Pintrich, P. R. (2000). The role of goal orientation in self-regulated learning. En: M. Boekaerts, P. R. Pintrich \& M. Zeidner (Eds.) Handbook of self-regulation (pp. 452-502). New York: Academic Press.

Ricardo, A. F., Mata, L., Monteiro, V. \& Peixoto, F. (2012). Motivação para a aprendizagem da matemática e sua relação com percepção de clima de sala de aula. En: Atas do Colóquio Internacional de Psicologia e Educação (pp. 1153 - 1168). Lisboa. .

Rosário, P. S. L., Núñez, J. C. \& González-Pienda, J. (2006). Comprometer-se com o estudar na universidade: cartas do Gervásio ao seu umbigo. Coimbra: Edições Almedina, S.A.

Rosário, P. S. L., Veiga Simão, A. M., Chaleta, E. \& Grácio, L. (2008). Auto-regular o aprender que espreita nas salas de aula. En. M. Abrahão (Ed.) Professores e alunos: aprendizagens significativas em comunidades de prática educativa (pp. 115-132). Porto Alegre: EDIPUCRS.

Rosário, P. S. L. (2013). Aprendizagem: processos de conhecer, metaconhecer, aprender e resolver problemas. En: F. H. Veiga. (Ed.). Psicologia da educação: teoria, investigação e aplicação. Envolvimento dos alunos na escola (pp. 297 - 332). Lisboa: Climepsi Editores.

Schunk, D. (1998). Teaching elementary students to self-regulate practice of mathematical skills with modeling. En: D. Schunk \& B. Zimmerman (Eds.). Self-regulated learning: from teaching to self-reflective practice (pp. 137 - 159). New York: The Guilford Press.

Shih, S. \& Alexander, J. M. (2000). Interacting effects of goal setting and self-or other-referenced feedback on children's development of self-efficacy and cognitive skill within the Taiwanese classroom. Journal of Educational Psychology, Vol. 92, p. 536-543.

Sousa, T., Monteiro, V.; Mata, L. \& Peixoto, F. (2010). Motivação para a Matemática em alunos do Ensino Secundário. En: Atas do Simpósio Nacional de Investigação em Psicologia (p. 2805 - 2819). Portugal, Universidade do Minho.

Souza, L. F. N. I. (2006). Crenças de auto-eficácia matemática. En: R. G. Azzi \& S. A. J. Polydoro (Eds.). Auto-eficácia em diferentes contextos (pp. 111 - 126). Campinas, SP: Editora Alínea.

Souza, L. F. N. I. \& Brito, M. R. F. (2008). Crenças de autoeficácia, autoconceito e desempenho em Matemática. Estudos de Psicologia, Campinas, No .25 , Vol. 2, p. 193-201.
Souza, L. F. N. I. (2010). Estratégias de aprendizagem e fatores motivacionais relacionados. Revista Educar, Curitiba: Editora UFPR, No. 36, p. 95 - 107.

Souza, L. F. N. I. (2013). Atitudes, concepções, crenças e aprendizagem matemática. En: Atas do Encontro Nacional de Educação Matemática, Curitiba, Paraná.

Torisu, E. M. (2010). Crenças de autoeficácia e motivação para matemática: um estudo com alunos do Ensino Fundamental de uma escola pública de Ouro Branco/MG. Dissertação (Mestrado em Educação Matemática) - Instituto de Ciências Exatas e Biológicas, Universidade Federal de Ouro Preto, Ouro Preto.

Torisu, E. M. (2012). Acompanhamento extraclasse e fortalecimento das crenças de autoeficácia matemática. En: Atas do Seminário Internacional de Pesquisa em Educação Matemática (pp. 1 - 22). Petrópolis, Rio de Janeiro.

Veiga Simão, A. M. (2006). Auto-regulação da aprendizagem: um desafio para a formação de professores. En: R. Bizarro \& F. Braga (Eds.). Formação de professores de línguas estrangeiras: reflexões, estudos e experiências (pp. 192 - 206). Porto: Porto Editora.

Veiga Simão, A. M. \& Flores, A. (2007). Using interviews to enhance learning in teacher education. Proceedings of the 52nd ICET World Assembly ICET and 6th Annual Border Pedagogy. En: Atas da Conference Borders, Boundaries, Barries and Frontiers: Promoting Quality in Teacher Education. San Diego, Califórnia, USA.

Yin, R. (2010). Estudo de Caso: planejamento e métodos. Porto Alegre: Bookman.

Zimmerman, B. J. (2000). Attaining self-regulation: a social cognitive perspective. En: M. Boekaerts, P. Pintrich \& M. Zeidner (Eds.). Hanbook of Self-regulation (pp. 13-39). New York: Academic Press.

Zimmerman, B. J. \& Campillo, M. (2003). Motivating self-regulated problem solvers. En: J. E. Davidson \& R. J. Sternberg (Eds.). The psychology of problem solving (pp. 233-262). Cambridge, UK: Cambridge University Press.

Zimmerman, B. J. (2013). From cognitive modeling to self-regulation: a social cognitive career path. Educational Psychologist, New York, $\mathrm{N}^{\mathrm{o}} .3$, Vol.48, p.135-147.

\section{Agradecimento}

Este trabalho é financiado pela CAPES. 